\title{
Lunxu Liu: a decade of dedication makes a leader in thoracic surgery
}

Submitted Apr 20, 2018. Accepted for publication Apr 26, 2018.

doi: $10.21037 /$ jtd.2018.05.02

View this article at: http://dx.doi.org/10.21037/jtd.2018.05.02

\section{Expert's introduction}

Prof. Lunxu Liu is the Vice President of West China Hospital \& Vice Dean of West China School of Medicine of Sichuan University, professor, chief physician, doctoral tutor, Fellow of RCS, AATS membership, and winner of the honorable title of "National Young and Mid-age Expert with Outstanding Contributions to Health and Family Planning" (Figure 1). Currently he also serves as Vice President of Chinese Association of Thoracic Surgeons and the Chairman of Expert Committee of Minimally Invasive Thoracic Surgery of Chinese Association of Thoracic Surgeons, the Standing Committee member of Chinese Society for Thoracic and Cardiovascular Surgery and the head of Thoracoscopic Surgery Group of Chinese Society for Thoracic and Cardiovascular Surgery, the Committee member of Chinese Anti-cancer Association on Lung Cancer, Vice Chairman of the Expert Committee of Thoracic Surgery of Chinese Research Hospital Association.

He took a lead in China in the introduction of complete video-assisted thoracoscopic surgery (VATS) radical treatment for lung cancer. In the international arena, he developed the "single-direction VATS lobectomy", and was the first surgeon to treat central lung cancer by performing complete VATS bronchovascular double sleeve lobectomy. He developed the non-grasping en bloc mediastinal lymph node dissection (MLND), "suction-compressing angiorrhaphy technique (SCAT)" for thoracoscopic massive hemorrhage, and the comprehensive treatment strategies for complex anatomy of hilum. Also, he performed the first successful bilateral lung transplantation in western region of China. He has won the Chinese Medical Sci-Tech Advance Award (first class) and Sichuan Provincial Science and Technology Advance Award (first class).

I first knew Prof. Lunxu Liu in my school days, when he already enjoyed a national reputation. I later learned more about him at various academic meetings. He is unassuming and taciturn, but difficulty-defying and efficient in action,

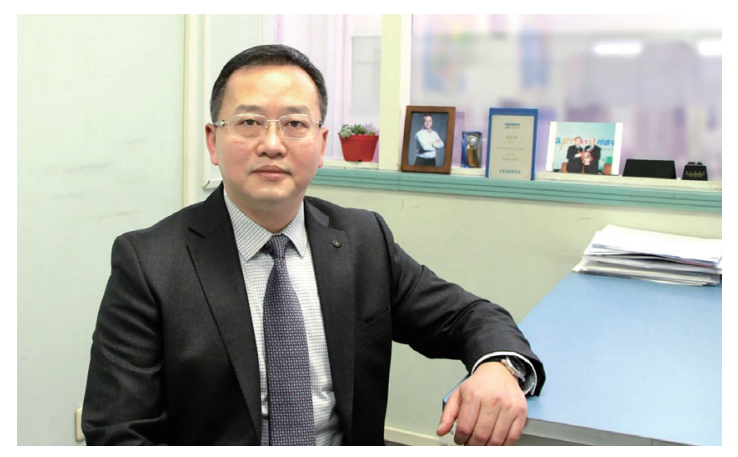

Figure 1 Prof. Lunxu Liu.

which is characteristic of Chongqing natives. Practicing as a doctor for nearly three decades, he invented "singledirection VATS lobectomy" and established a steady leadership in medical technology. He said, "As doctors, we should not only love the career but also keep exploring better solutions to clinical problems to promote the development of medical science".

\section{Respect for doctors leads to career success}

Prof. Liu was born in Chongqing and set ambitious goals when he was very young. "I believed doctors and teachers are noble professions, and I chose to study medicine because it's about death and life." That was the primary reason behind Liu's career choice. In a time when the Chinese economy had yet to take off, it was the respect for medical science and education that propelled him to indulge in the research of minimal invasion technology. As a doctor, Prof. Liu has saved numerous patients from death; as a teacher, he spares efforts to overcome formidable medical challenges together with his students.

Lunxu Liu graduated in 1989 from West China University of Medical Science (now West China Medical Center, Sichuan University) and continued to work in the university. In order to learn more advanced technologies, 
he became a full-time graduate student in 1992. In 1997, he returned to West China Hospital as a thoracic surgeon after obtaining a Doctoral degree.

Prof. Liu was trained in Australia twice, which has a profound influence on his later commitment in minimally invasive surgery. "We should first know how our foreign counterparts do it". He said it was better to read ten thousand books and travel ten thousand miles, and seclusion from the outside world was unfavorable to technological innovation and development. "One decade ago, many foreign concepts and technologies outstripped domestic ones and provided guidance for our development. Aside from learning techniques, I paid more attention to the whole process of diagnosis and treatment and medical theories, which was more inspiring to me".

\section{Dedicated to single-direction VATS lobectomy}

The invention, development, and popularization of a new technology usually have to come through zigzags. VATS is no exception.

VATS is the most important step forward in thoracic surgery in the late 20th century. The world's first VATS resection of lung cancer was successfully performed in 1992, but the technology stagnated for a long period of time after that. There were only a handful of successful surgeries in the world due to operational difficulties, demanding skills, and lack of efficient measures of hemostasis. Few Chinese doctors could perform this surgery. Some doctors even reckoned that complete VATS was not feasible for lung cancer.

However, Prof. Liu deemed that behind the seemingly insurmountable high mountain of VATS lies a vast plain. "I was absolutely convinced that VATS would be the future trend as minimally invasive technique that would benefit more patients. The faith motivated my persistent exploration". Why was such a good technology difficult to be implemented? "The method must be problematic, and we need to find a way to improve the surgery and facilitate its implementation". Prof. Liu was therefore committed to exploring new methods and approaches of VATS.

The big challenge inspired Prof. Liu to innovate. He slept only 3 to 4 hours a day. Aside from clinical practices and administrative affairs, he spent all the rest time on new technology research. He collected relevant information on the Internet and in the library, watched and thought closely in daily lung cancer surgeries in order to conduct further studies in human-body models and specimens.

Based on the massive amount of clinical data in West
China Hospital, Prof. Liu found that the thoracoscopic incisions in the past were mainly centralized on the lateral chest wall, and the operation angle was difficult to control; when the instruments entered the thoracic cavity, they just pointed to the heart and thus might easily cause heart and vessels damage. Also, the pulmonary lobes need to be turned back and forth during the operation, leading to high incidences of accidental injuries and high rate of conversion to thoracotomy. In addition, thoracoscopic surgeries required multi-point dissection, during which multiple instruments are needed, which made the operations even more difficult. Through repeated explorations, Prof. Liu changed the locations of the incisions to "vertical-parallel" style, and developed the single-site single-direction approach that was only allowed operations at lung hilum rather than inside lungs and performed layer by layer from superficial to deep; this single-direction resection could both simplify the operation and achieve ideal therapeutic effectiveness. He put his whole self into the research, working night and day. Finally, the single-direction VATS lobectomy was successfully developed and applied in clinical settings.

In 2006, an elderly frail patient with lung cancer became the first beneficiary of this procedure. An open surgery with large incisions was too risky for this patient, who might be unable to make it for recovery. Single-direction VATS lobectomy offered the patient an alternative. Prof. Liu admitted that he and his team were under huge pressure before the surgery. "At the time, minimally invasive surgeries, especially VATS resection of lung cancer, had yet to be widely recognized for fear of accidents and failure to remove the tumor completely". Fortunately, West China Hospital encouraged young doctors to seek breakthroughs and gave strong support for his team. Meanwhile, Prof. Liu was highly confident about the surgery based on previous exploration and experiences. The surgery proceeded smoothly. Although it was the first operation of this kind, it didn't take too much time and the patient recovered well. Based on massive studies, this procedure was finally established. "You might say that this procedure was created due to clinical demand," said Prof. Liu.

Prof. Liu and his team rose to fame thanks to the innovation, but he did not name the procedure by himself. He said, "It is more objective to name the surgery through the technology and easier to promote the procedure. 'Single direction' can better explain its technological feature. Besides, unlike in foreign countries, it is not a convention in China to name a procedure after the developer's surname". 


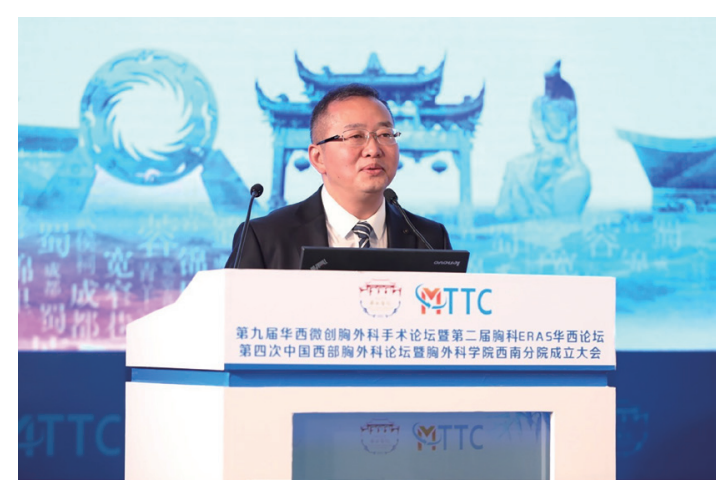

Figure 2 Prof. Lunxu Liu gives lectures at the Ninth West China Hospital Forum on Minimally Invasive Thoracic Surgery \& the Second West China Hospital Forum on Thoracic ERAS. ERAS, enhanced recovery after surgery.

\section{Introduce the "single-direction" procedure to the world}

As the new procedure matured, Prof. Liu and his team started to promote it.

In 2007, the Thoracic Surgery Department of West China Hospital hosted the "First West China (International) Forum on Minimally Invasive Thoracic Surgery". Prof. Liu's team demonstrated the single-direction VATS lobectomy at the forum. "After watching our demonstration, the attendees realized that VATS could be performed so easily, and such problems as uncertain effectiveness and low efficiency of VATS lobectomy could be solved in this way". The participants were excited by the demonstration. Many thoracic surgeons learned the procedure and began to apply it at their own hospitals, finding that it was easier to master than traditional approaches. High recognition from the peers enabled the technology to be rapidly popularized nationwide.

Prof. Liu's team also established the West China VATS Lobectomy System, which includes common VATS lobectomy, difficult/complicated lobectomy, and management of accidents. Finally, the single-direction VATS lobectomy becomes a mainstream procedure in thoracic surgery for lung cancer. Nearly $90 \%$ of lung surgeries in West China Hospital have adopted this technique.

Prof. Liu is pleased that this procedure plays a guiding role. "Many technological problems have been solved through this procedure or its derived technologies such as dissection of lymph nodes, management of accidental massive haemorrbage, and troubleshooting of difficult freezing lung hilum. In addition, we performed the world's first VATS bronchovascular double-sleeve lobectomy for central lung cancer. In fact, we have created a whole set of VATS solutions for lung cancer".

Aside from lung cancer surgeries, esophageal cancer surgeries in West China Hospital also adopt minimally invasive technology. Prof. Liu said new methods were used in video-assisted minimally-invasive surgeries for esophageal diseases. For instance, advanced concepts of improving the function of gastric conduit and reducing complications such as anastomotic fistula have facilitated the performing of surgeries with better outcomes. Each thoracic surgeon at the hospital has mastered minimally-invasive technology. Under the guidance of Prof. Liu, young surgeons have kept innovating. Dr. Hu Yang won the first prize in the videoassisted esophageal surgery group in the "2017 Fourth Elite Cup National Surgical Skill Competition for Outstanding Young Thoracic Surgeons".

Since 2007, West China Hospital has organized ten editions of "West China (International) Forum on MiniInvasive Thoracic Surgery", 24 VATS classes, and trained more than 6,000 surgeons, 65 doctorate candidates and 81 master candidates (Figure 2). Prof. Liu has been invited to give lectures in 16 international academic meetings and more than 150 domestic ones, and performed over 30 live surgeries at domestic academic conferences and the Annual Meeting of Asian Society for Cardiovascular Surgery. The single-direction VATS lobectomy has so far been adopted by nearly $90 \%$ of tertiary hospitals in 31 provinces, autonomous regions and municipalities.

Good wine needs no bush. The single-direction VATS lobectomy has drawn increasing attention from the international medical community. Prof. Liu is one of the Royal College of Surgeons (RCS) fellows in the field of thoracic surgery as well as a member of the American Association for Thoracic Surgery (AATS). In November 2014, the Minimally Invasive Surgery Training Center of West China Hospital passed the RCS accreditation. Prof. Liu said, "We are glad to be awarded with these honors, which also mean greater responsibilities and obligations. First of all, we should enhance communications with domestic and foreign colleagues, especially those in the US, which leads in thoracic surgery. Secondly, we should publicize China's advantages. It's time for "exporting" our technology. We have become leaders in some sectors and should undertake corresponding responsibilities".

Combining West China Hospital Thoracic Surgery Department's experiences and RCS's scientific and sophisticated curriculum, Prof. Liu sponsored the "International VATS Training Program (Europe)" for a number of senior doctors from six medical centers in 
Germany, Britain, and the Netherlands. Unlike previous international programs focusing on theory exchanges, this training program was systematic and offered the trainees with training materials. Interaction between teachers and learners, collection of opinions and feedback from trainees upon completion, and tracking of the learning effect were added to realize the goal of this systematic training. "In the past, trainers could hardly know the learning efficacy, followingup activities, and difficulties of trainees after the classes. Now we create a closed loop via standard curriculum and rigorous feedback to improve the training effect". Prof. Liu said, "So it is a genuine training program. China's thoracic surgery technologies can be systematically introduced to other countries via this way, deepening foreign experts' understanding of the status quo of thoracic surgery in China".

\section{Enhanced recovery after surgery (ERAS) in West China Hospital: doing proper addition and subtraction for patients}

According to Prof. Liu, minimally invasive surgeries should move from training and learning to the establishment of diagnosis and treatment guidelines based on current practices. Some new and cutting-edge technologies such as ERAS, precision medicine, artificial intelligence, and big data should be incorporated into thoracic surgery to form a multidisciplinary "minimally invasive environment".

At present, the Department of Thoracic Surgery of West China Hospital is still leading the development of ERAS in the Southwest China and even across the whole country. ERAS requires a series of measures taken in perioperative period to reduce the stress response, so as to achieve rapid physiological rehabilitation. It is an inevitable result of the advances in medical theory and surgical technology, reflecting the concept of "patient-centered care".

"The concept of ERAS bas been put forward for a long time, but its application in thoracic surgery is lagging behind." Since ERAS has challenged the traditional concepts of some textbooks and guides, it may face risks in practice. Thus, it needs the advocation and promotion at hospital and department levels before medical staff and patients can accept these new concepts and technologies. West China Hospital encourages innovations, especially those welljustified and evidence-based ones. The hospital attaches great importance to ERAS, which has been forcefully promoted at both hospital and department levels.

In addition, ERAS is a systematic project which requires multidisciplinary collaboration. Unlike multiple disciplinary team (MDT), it has wider coverage and requires the optimization of all processes before, during, and after surgery. According to Prof. Liu, West China Hospital again takes a lead in ERAS, which owes to its harmonious and mutually inclusive culture that eliminates the difficulties and obstacles of multidisciplinary collaboration.

"Therefore, the success of ERAS in West China Hospital should be credited to its organizational culture and to the leadership of the hospital." said Prof. Liu.

For ERAS, Prof. Liu and his team always insist on doing proper addition and subtraction for patients.

"Addition" means the use of more useful measures for the recovery of patients during the perioperative period, so as to increase the accuracy of disease diagnosis/assessment, lower the risk of the operation, and reduce the incidences of complications. Second, they try to refine the principles and measures of surgical indications; for instance, more sophisticated diagnostic tools (e.g., precision medicine at molecular level) are used to increase the accuracies in disease diagnosis and treatment. Finally, by offering psychological counseling and supporting rehabilitation, they attempt to speed up the rehabilitation and help patients to return to life and work earlier.

"Subtraction" is to reduce unnecessary operations that cause extra trauma to patients. It may involve the adoption of minimally invasive technique, reduction in procedures, and shortening of hospital stay. While some of these measures were regarded as "required" in past, reducing these operations can actually benefit patients during ERAS.

"In the fields of ERAS and airway management, however, while some definite evidences have been available, other findings remain controversial." Said Prof. Lunxu Liu, "We need to carry out more multicenter clinical studies to obtain high-level evidences to support the concept of ERAS and to justify both 'addition' and 'subtraction"'.

Prof. Liu is full of confidence for the future of ERAS, just as he is so confident about single-direction VATS lobectomy. "Any promising technology will for sure be promoted. The difficulty existed in a technology may also be the key point to break through. A sharp knife may damage blade when it meets a bone. However, once the bardest site is dissected, it will be easy to cut the whole bone open."

\section{Allocating time and energy in a reasonable manner}

In September 2017, Prof. Liu was appointed Vice President of West China Hospital. In capacities of vice president, 
department director and thoracic surgeon, he has very little spare time but never lowers requirements on himself. "Each stage of life has its most important things. We must do these things well first and then embark on other things to save time, for the sake of doing a better job."

According to Prof. Liu, efforts made in off-work hours reflect his team's vigor and devotion to medical development. "As a team, sometimes we would discuss a hot topic or research from 7:00 pm to 1:00 am." He often exhorted young doctors, "If you really want to make the progress, extra efforts should be made outside the 8 working hours. Don't hasten to gain but be patient to learn and pay".

In face of administrative affairs, medical practices and scientific researches, Prof. Liu applied the experience of ERAS in time management. He revealed that his time is scheduled in the unit of 30 minutes. Each time unit has an item. Besides, as vice president of the hospital, he faces many unexpected events. In order to do each thing well, it is necessary to manage time and balance work by allocating time and energy in a reasonable manner.

Prof. Liu is so devoted to his work that he spends little time with his family. "I didn't expect to be so busy with work, but the work burden accumulated gradually, and that gave me more time to learn how to manage time. But family life was somehow neglected." He said in an apologetic tone, "My family members understand and support me, though they complain sometimes". In order to compensate his absence in family life, he strives to make trade-offs between work and family and strike a balance.

Based on his experience as a doctor for three decades, Prof. Liu summed up four principles for young doctors: coming down to earth, having foresight, being committed

Cite this article as: Leng $\mathrm{X}, \mathrm{Li}$ P. Lunxu Liu: a decade of dedication makes a leader in thoracic surgery. $\mathrm{J}$ Thorac Dis 2018;10(Suppl 11):S1308-S1312. doi: 10.21037/jtd.2018.05.02 to public credibility, and being tenacious. He reckoned that it is the key to success to do things in a surefooted manner; foresight is an important driving force of sustainable development, and one should keep a low profile while being farsighted; perseverance will eventually pay off. Besides, "rules" should be viewed from different angles. Innovation may deviate from intended results without compliance to established rules, whereas putting rules above all could lead to dogmatism. Therefore, innovations should be sought without breaking the rules.

Prof. Liu is both a dreamer and a doer in his careers. He keeps innovating and putting novel ideas into practice, and pursues his dream step by step to live a successful and colorful life.

\section{Acknowledgements}

We wish to express our gratitude to L Liao and C Gao of the AME Publishing Company for their strong support and assistance for this article and to Q $\mathrm{Li}$ of the AME Publishing Company for his support and assistance with the interview.

\section{Footnote}

Conflicts of Interest: The authors have no conflicts of interest to declare.

(Section Editor: Xuefeng Leng, Department of Cardiothoracic Surgery, Affiliated Hospital, Chengdu University, Chengdu 610081, China; Science Editor: Pengcheng Li, JTD, jtd@amepc.org) 